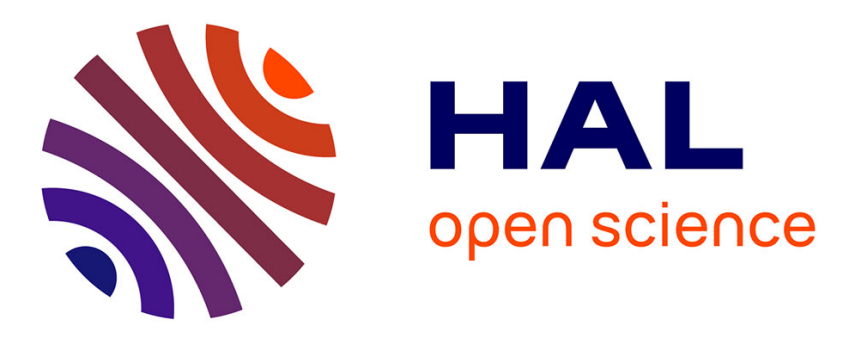

\title{
A Wearable LoRa-Based Emergency System for Remote Safety Monitoring
}

\author{
Gaby Bou Tayeh, Joseph Azar, Abdallah Makhoul, Christophe Guyeux, \\ Jacques Demerjian
}

\section{- To cite this version:}

Gaby Bou Tayeh, Joseph Azar, Abdallah Makhoul, Christophe Guyeux, Jacques Demerjian. A Wearable LoRa-Based Emergency System for Remote Safety Monitoring. International Wireless Communications and Mobile Computing Conference, Jun 2020, Limassol, Cyprus. hal-02993852

\section{HAL Id: hal-02993852 \\ https://hal.science/hal-02993852}

Submitted on 7 Nov 2020

HAL is a multi-disciplinary open access archive for the deposit and dissemination of scientific research documents, whether they are published or not. The documents may come from teaching and research institutions in France or abroad, or from public or private research centers.
L'archive ouverte pluridisciplinaire HAL, est destinée au dépôt et à la diffusion de documents scientifiques de niveau recherche, publiés ou non, émanant des établissements d'enseignement et de recherche français ou étrangers, des laboratoires publics ou privés. 


\title{
A Wearable LoRa-Based Emergency System for Remote Safety Monitoring
}

\author{
Gaby Bou Tayeh ${ }^{a}$, Joseph Azar ${ }^{a}$, Abdallah Makhoul ${ }^{a}$, Christophe Guyeux ${ }^{a}$ and Jacques Demerjian ${ }^{b}$ \\ ${ }^{a}$ Femto-St Institute, UMR 6174 CNRS, Université de Bourgogne Franche-Comté, France \\ Emails: gaby.bou_tayeh@univ-fcomte.fr; joseph.azar@univ-fcomte.fr; abdallah.makhoul@univ-fcomte.fr; \\ christophe.guyeux@univ-fcomte.fr \\ ${ }^{b}$ LaRRIS, Faculty of Sciences, Lebanese University, Fanar, Lebanon \\ Email: jacques.demerjian@ul.edu.lb
}

\begin{abstract}
With the advent of the industrial revolution, human beings have developed drastically over the past decades. By 2020 , wireless communications would connect more than twenty-five billion devices. Low Power Wide Area (LPWA) technologies are becoming popular as a result of the fast development of the Internet of Things (IoT) market. In this paper, we propose a wearable LoRa-based system for remote safety monitoring of people performing activities in remote areas with no network coverage. The designed system is supposed to detect possible heart problems and/or a "man-down" situation. It then transmits an emergency alert containing information about the state of the concerned individual and its location via LoRa to the surrounding recipients. The proposed system composed of a GPS enabled IoT device, a smart-watch and a smart-phone, has been validated in a remote area in the city of Belfort in France. The obtained results demonstrate the feasibility of such a system.
\end{abstract}

Index Terms-LoRa, LPWAN, Wireless Sensor Networks, IoT, Healthcare

\section{INTRODUCTION}

In recent years, the LoRa (Long Range) and LoRaWan (Long Range Wide-area network) technologies have emerged as the new de facto of providing Wireless connectivity between a network of sensors. Numerous services and applications have been built around them, motivated by the vast benefits and advantages the LPWAN (Low Power Wide Area Network) technology provides, such as their ability to ensure services that cover a large area of multiple kilometers, they could be implementable on devices powered by low energy resources, they provide a practical and low deployment cost. One of the domains that have significantly benefited from these technologies is the healthcare domain. The capability of transmitting data over a long distance while consuming a low amount of energy paved the way for various applications and services to be developed.

\section{A. Background}

Lora wireless networks made it possible to monitor poor and dangerous environmental conditions. Applications for safety and environmental monitoring involve efficient, durable, fast response and reliable systems. By introducing a self-powered wearable IoT sensor network that gathers and sends environmental information to the cloud through a LoRa gateway, the authors in [1] proposed a solution for individuals working in difficult environments. The suggested scheme utilizes LoRa technology for wireless transmission to provide the network with a long-range and low-power solution. In [2], the authors present a LoRaWan based health monitoring system aimed to transmit sensor data of patients living in rural areas to a central medical center in order to reduce the burden of having to travel to distant facilities to have them examined. The authors in [3] developed a Wireless Body Area Network (WBAN) aimed to improve the safety of construction workers. The latter are equipped with body sensors that collect information such as temperature, humidity, UV, $\mathrm{CO} 2$, heart rate, and body temperature. The sensors can communicate with each other to exchange information using BLE, and a dedicated safe node transmits the information through a gateway to a cloud server using the LoRaWan network protocol. In [4], the authors assessed LoRa LPWAN technology for indoor remote health and drew the following findings: 1) The feasibility of LoRaWAN for healthcare applications and indoor communication. 2) Indoor communication connections of over 300 meters are feasible, the communication performance varies strongly and is affected by the equipment and barriers blocking the link. 3) The maximum spreading factor does not always give the most stable communication link. 4) There is always the possibility that packets will be lost regardless of the communication settings used. 5) LoRaWAN is more appropriate for applications that are tolerant to delay and loss.

LoRa's extensive work shows that it has excellent potential for deployment in both indoor and outdoor physical environments as well as monitoring individuals in hazardous environments.

\section{B. Motivation and problem formulation}

It has recently become an important task to monitor individuals doing activities or working in risky outdoor settings. Hiking, camping, biking, skiing or other outdoor leisure can lead to a risk of injuries or even death. Even a small accident may be dangerous in case it happened in a remote place or during extreme weather conditions that make it difficult for the injured individual to seek help. The risks that come with recreation activities are numerous such as, slipping on rocks, 
logs when crossing bodies of water, become separated from the group, getting lost and run out of food and water. other risks involve dehydration, hypothermia, heatstroke, frostbite, back injury, and many others.

For instance, on September 11, 2019, a story went viral on multiple US news outlets. It happened in Arroyo Seco River, where two men found a bottle with a note inside "we are stuck, we are at the waterfall, get help" [5]. They then hiked a couple of miles to alert the authorities. A rescue team went to look for potential victims and eventually found a family stuck at the Arroyo Seco narrows since the waterfall they needed to cross was too strong. Unfortunately, this is not the first and the last story of this kind and this is why multiple studies [6] have been conducted to understand the risk of injuries during such activities. For example, as cited in [7], from 1998 to 2011, the Rocky Mountain Rescue Group (RMRG) rescued 2,198 mountain and wilderness victims in Boulder County, Colorado. Rock climbing victims were 428 or $19.5 \%$ of all victims. According to the Système National d'Observation de la Sécurité en Montagne (S.N.O.S.M) in France, since 2012 5,389 interventions of mountain rescue services have been conducted, 172 resulted in deaths and disappearances, as well as 3,385 injured were reported (excluding resort ski areas). Moreover, according to a study published in [8], taking the 5-year average for 2008 to 2012, annually 60,000 skiers and 17,000 snowboarders on Swiss slopes are injured seriously enough to require medical attention. Moreover, every year 19 people on average died while skiing or snowboarding, whereas the numbers are significantly higher in other countries such as Greece and the US.

This paper focuses on healthcare and outdoor environmental applications. The targeted issue of this work can be formulated as follows. How can LPWAN technologies help track the location and monitor the health conditions of individuals performing activities in distant, dangerous, and non-covered areas with no network access?

\section{Contribution}

This paper presents a complete system that includes both a hardware and a software solution for the previously presented problematic.

- Hardware: We propose a network architecture composed of three main components that enable the transmission of an alert from a source to a destination over a long distance without the need for GSM coverage. The devices composing the system are unobtrusive (smart-watch, smartphone) and used by the users in their daily life. In addition, The IoT device responsible for transmitting messages with LoRa is tiny and easy to mount and does not cause any discomfort. Moreover, such devices are low cost which makes the proposed system affordable for its users.

\section{- Software:}

We propose a system that transmits an emergency alert automatically to the surrounding recipients or to the group to which a person belongs in the event of an emergency. Moreover, it allows its localization, which could save time and effort to find the individual and can potentially save his life. To do so, on the smart-watch, two algorithms were introduced, the first automatically detects a heart problem and the second automatically detects a mandown state (caused by a fall, knee or ankle break, fainting, etc.).

\section{Organization}

The rest of the paper is organized as follows: Section II discusses the design and components of the proposed system. Section III presents the software implementation of the algorithms used for emergency detection. Section IV discusses the implementation of the proposed system. Section V discusses the energy consumption and the coverage of the system and Section VI concludes the paper.

\section{DESIGN AND COMPONENTS OF THE PROPOSED SYSTEM}

In this section, we will describe the proposed system, illustrate its design, and list and explain the role of each of its components. We will first start by briefly explaining the objective of our proposal. As previously mentioned in Section I-B, a significant number of people are at risk of getting injured while performing recreational activities in remote areas. In case of severe injuries and the need for a dedicated rescue team to intervene, the rapidity of notifying this team is a matter of life and death. In a remote area with no GSM coverage, it requires a person to physically head to the rescue personnel that could be located far away in order to notify them that someone is a need of help. Nonetheless re-localizing this person is not an easy task as well. This puts the injured person in great danger. The worst scenario is when someone loses his group, ends up alone and faces an incident, gets injured, faint, etc. It would be impossible for him to notify someone, his only chances are that he gets sighted by someone that could summon the rescue team for him.

In order to resolve these crucial issues, we propose a LoRa based emergency alert system. The proposed system is designed to transmit an alert that includes the GPS location to the surrounding recipients in case of emergency, they could be other nearby people equipped with this system or the rescue command station that is always listening to incoming alerts. The system can detect a heart problem (stroke, abnormal heartbeat) as well as when the wearer of the system stops moving. In both cases, it automatically transmits an alert. The wearer of the system could also manually trigger an alert in case he needed help.

\section{A. The system's components}

The first component of the system is the Pytrack sensor shield (Figure 1a). This device is equipped with a super accurate GNSS Glonass GPS and it supports the following location services: GPS, GLONASS, Galileo, and QZSS. It has a relatively small size of $55 \mathrm{~m} \times 35 \mathrm{~mm} \times 10 \mathrm{~mm}$ and it weighs $11 \mathrm{~g}$. This device will be used to acquire the GPS location of the individual in case of an emergency.

The second component of the system is the LoPy 4 development board (Figure 1b). This device is equipped with Semtech 
LoRa transceiver SX1276 that has a transmission range that can reach up to $40 \mathrm{Km}$ in ideal conditions. The device has a small size of $55 \mathrm{~mm} \times 20 \mathrm{~mm} \times 3.5 \mathrm{~mm}$ and it weighs $7 \mathrm{~g}$. The algorithm that acquires the GPS location in case of emergency and broadcast the alert will be embedded in this device. The LoPy4 will be attached to the Pytrack using pins which enables it to give instruction commends to the latter and exchange data.

Fig. 1: Components of the GPS enabled IoT device

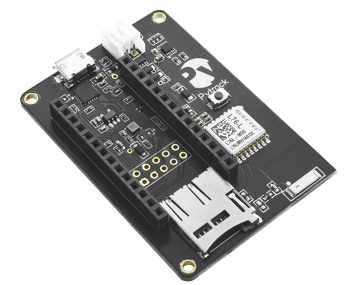

(a) Pytrack sensor shield

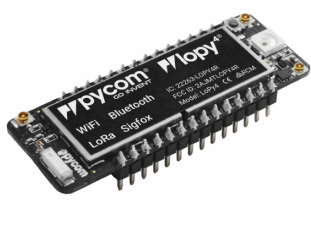

(b) The LoPy4
The third component of the system is the smart-watch. In our case, we have used the Huwaei watch 2 and the Polar M600. Both are equipped with a 3-axis $A+G$ sensor that will be used to detect the activity of the watch wearer. It also includes a heart rate sensor (PPG) that will be used to collect heart rate measurements and detect any abnormal activity.

Finally, the last component is the smart-phone. The latter is useful for the alert receivers, it displays on the screen the cause of the emergency alongside the location and the direction to the emitter. This enables the recipients to easily localize a victim.

Fig. 2: Network composition

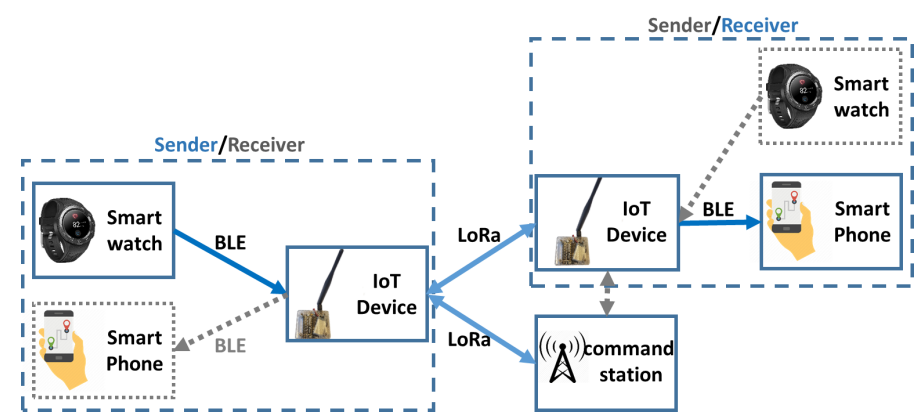

\section{B. The design of the proposed system}

In this section, we will explain in depth the functionalities of each component, where it belongs, its role in the hierarchy of the network, and how these different components are interconnected in order to transmit an alert from a source to a destination.

As mentioned earlier, the wearable system is composed of three main devices, the smart-watch, the smart-phone, and the GPS enabled IoT device (Pytrack + LoPy4). The IoT device is associated and connected to the smart-watch and the smartphone via Bluetooth Low Energy (BLE).
The smart-watch: Using its integrated accelerometer and heart rate sensors, it can detect whether the watch wearer has stopped moving for a period of time, or he has a heart problem.

The IoT Device: Acquire the GPS coordinates of the system wearer and broadcast an alert using its integrated LoRa transceiver.

The smart-phone: Display the received alert alongside the location of the emitter.

Figure 2 illustrates how the system works. Each individual is equipped with an IoT device, a smart-watch, and a smartphone, and it can act as an alert sender or a receiver.

Case Sender: Either the Watch automatically detects an emergency or the watch wearer manually triggers an alert. A status description message (e.g.: not moving, heart problem, lost, etc.) is then transmitted via BLE to the IoT device. The IoT device acquires the GPS coordinates, adds the coordinates to the payload, and broadcasts the message via LoRa and waits for an acknowledgment. It keeps re-transmitting the alert every few seconds (e.g.: 30 secs) as long as it did not receive any acknowledgment from nearby devices.

Case Receiver: The IoT device is always listening to upcoming messages. If received one, it first transmits an acknowledgment to notify the sender that his message has been detected. It then transmits the message via BLE to the smart-phone, which in turn alerts its user that someone is a need of help while providing its location. The receiver could also be a commend rescue station.

\section{SOFTWARE IMPLEMENTATION FOR EMERGENCY DETECTION}

This section first introduces the Early Warning Score (EWS) system used to assess the smart-watch pulse samples, then describes the emergency detection algorithms that utilize the EWS system and are implemented for emergency detection on the smart-watch.

\section{A. Early Warning Score System}

The use of an EWS system has long been enabled by acute care teams to allow a more auspicious response and assessment of patients who are highly sick or injured. An EWS system is based on a simple scoring scheme that assigns a score to each acute patient-monitored physiological measurement [9]. The EWS is used in this work to assess the heart rate samples gathered from the wearable device. For each vital sign, a normal healthy range is defined, and the score assigned reflects how extreme the measured value varies from the standard. The higher the score assigned, the more a collected value is outside the normal healthy range.

In this work, the scoring template for the National EWS (NEWS) used in U.K [10], illustrated in Figure 3, is used to assess the severity level of the pulse in order to detect an emergency situation.

\section{B. Algorithms implementation}

This section presents two algorithms to detect heart issues and a man-down state. Three kinds of sensors can be used 


\begin{tabular}{|c|c|c|c|c|c|c|c|}
\hline $\begin{array}{l}\text { Physiological } \\
\text { parameter }\end{array}$ & 3 & 2 & 1 & $\begin{array}{l}\text { Score } \\
0\end{array}$ & 1 & 2 & 3 \\
\hline $\begin{array}{l}\text { Respiration rate } \\
\text { (per minute) }\end{array}$ & $\leq 8$ & & 9-11 & $12-20$ & & $21-24$ & $\geq 25$ \\
\hline $\mathrm{SpO}_{2}$ Scale 1 (\%) & $\leq 91$ & $92-93$ & 94-95 & $\geq 96$ & & & \\
\hline $\mathrm{SpO}_{2}$ Scale $2(\%)$ & $\leq 83$ & $84-85$ & $86-87$ & $\begin{array}{c}88-92 \\
\geq 93 \text { on air }\end{array}$ & $\begin{array}{c}\text { 93-94 on } \\
\text { oxygen }\end{array}$ & \begin{tabular}{|c|}
$95-96$ on \\
oxygen
\end{tabular} & $\begin{array}{l}297 \text { on } \\
\text { oxygen }\end{array}$ \\
\hline Air or oxygen? & & Oxygen & & Air & & & \\
\hline $\begin{array}{l}\text { Systolic blood } \\
\text { pressure (mmHg) }\end{array}$ & $\leq 90$ & $91-100$ & $101-110$ & $111-219$ & & & $\geq 220$ \\
\hline Pulse (per minute) & $\leq 40$ & & $41-50$ & $51-90$ & $91-110$ & $\mid 111-130$ & 2131 \\
\hline Consciousness & & & & Alert & & & CVPU \\
\hline Temperature ( $\left.{ }^{\circ} \mathrm{C}\right)$ & $\leq 35.0$ & & $35.1-36.0$ & $36.1-38.0$ & $38.1-39.0$ & $\geq 39.1$ & \\
\hline
\end{tabular}

Fig. 3: National Early Warning Score (NEWS)

for such applications using contemporary wearable devices, namely the pulse sensor, motion sensor (accelerometer), and step counter sensor.

The first algorithm implemented on the wearable device is a periodic algorithm that runs after each period of time $t$ (Algorithm 1). The pulse and step counter sensors collect data on an ongoing basis and store the values in the memory. After each period $p$, the total number of steps taken between $p$ and $p-1$ in addition to the average heart rate values are used to detect an abnormal situation. Referring to [11], the average number of steps that can be taken in one minute for a low-intensity activity such as walking 3 miles per hour is 100. Having done less than 100 steps in a period with a pulse score of 3 (Figure 3) can reflect the possibility of an unusual situation such as injury or heat stroke. If the user has not done an extensive activity and yet has a pulse score of 3 , a notification with vibration will be displayed on the watch for a small amount of time to inform the user about his pulse rate. If he believes his pulse rate is normal and this is a false alarm, the user can dismiss the notification by just shaking his hand. If the user has not responded to the notification, an emergency message will be transmitted to the IoT device.

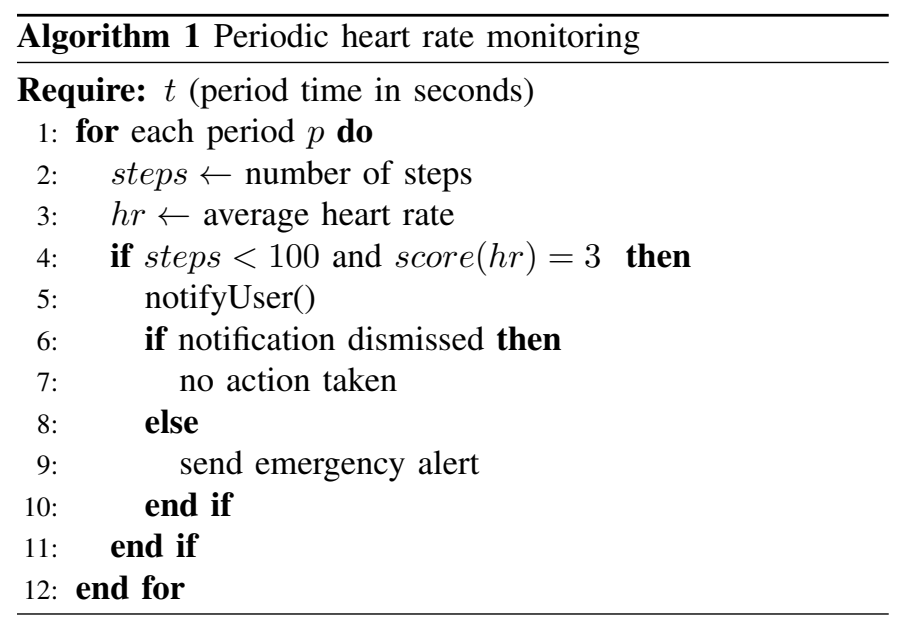

In order to detect a man-down state, Algorithm 2 has been implemented to run continuously in the background. This algorithm uses the accelerometer sensor to detect if the watch is not moving for a predefined amount of time. Same as for Algorithm 1, when the watch is not moving for more than $\beta$ amount of time, a notification with vibration will be displayed on the watch. If the user does not respond, an emergency message will be sent.

In addition to Algorithms 1 and 2, The user has the choice of sending an emergency message manually using the watch in case of loss.

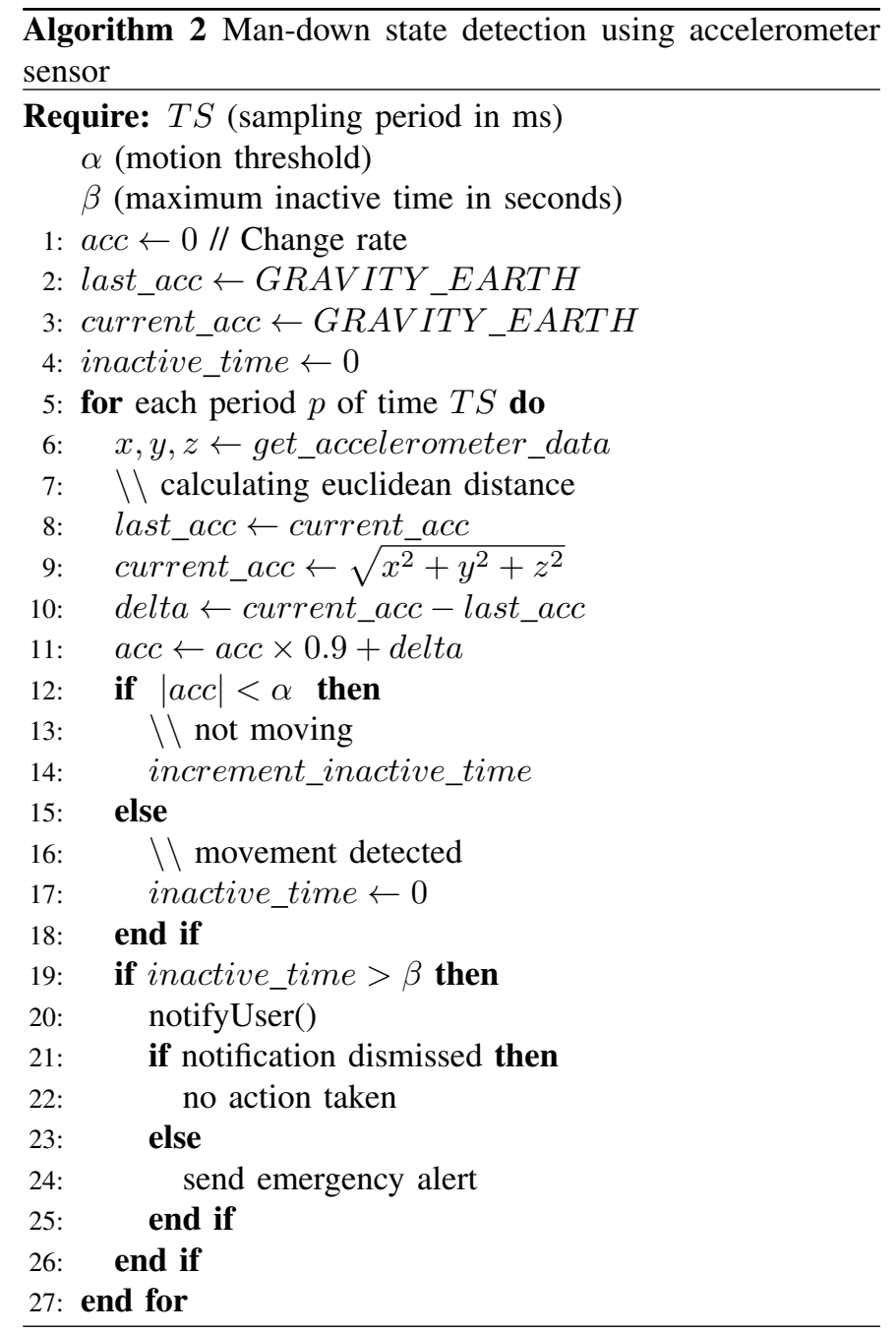

\section{VALIDATION AND VERIFICATION OF THE PROPOSED SYSTEM}

In this section, the results of an implementation of the system in an area surrounding a lake in the city of Belfort in France is presented. Three persons have been deployed in the testing area, each one of them was equipped with a watch, a smart-phone, and an IoT device. One of the three has been given the role of a victim (we will refer to this person as person $A$ ). The other two were deployed at approximately 1.2 $\mathrm{km}$ away from the supposed victim and waited for an alert (we will refer to them as $B$ and $C$ respectively). The equipment that has been used in this system validation experiment are 
shown in Figure 4 and a brief description of the LoRa settings we have used are listed below:

- the used radio frequency is $868 \mathrm{MHz}$;

- the LoPy4 Transmission power is set to $14 \mathrm{dBm}$;

- the loPy4 LoRa antenna has a 2dBi gain;

- the spreading factor (SF) is set to 12 ;

- the bandwidth (BW) is set to $125 \mathrm{KHz}$;

- the payload size of the alert packet is fixed to 8 Bytes.

With these settings, the time on-air (ToA) of our packet, which means the time it takes the packet to reach the receiver, will be approximately $631 \mathrm{~ms}$, which is very acceptable in case of emergency.

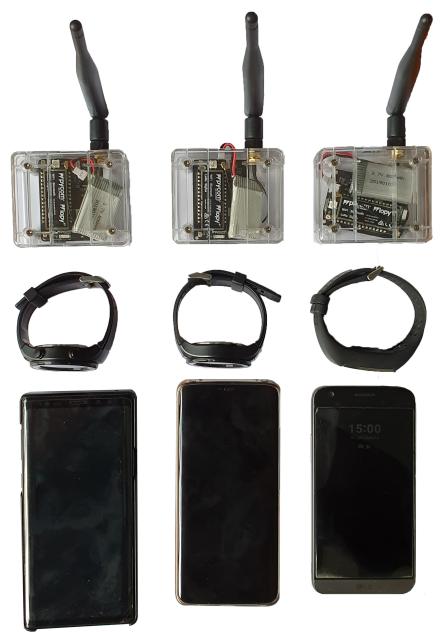

Fig. 4: The used equipement

In order to verify the Algorithm 2, person $A$ was asked to perform two tasks:

- standstill to simulate a "man down"state. This enables us to test whether the watch triggers an alarm and whether $B$ and $C$ at the other end are able to detect it and localize $A$;

- $A$ was given the ability to instantly increase his heart rate programmatically with a click of a button in order to simulate a heart problem. $A$ was asked to take a few steps only and increase his heart rate.

In both tests, an alarm was successfully detected by $B$ and $C$, and the location of $A$ has been reported.

Figure 5 illustrates the variations in the $a c c$ value described in Algorithm 2 in Section III-B. The latter identifies a mandown state if a certain period of time passes and no important changes in the acc value is detected. This can be seen in the red part of the example shown in Figure 5. A "man down" has been identified starting from sample number 5500 and above since no movement has been detected by the watch.

Figure 6 illustrates abnormal heartbeat detection. It can be seen that the average heart rate rises and can bypass $100 \mathrm{bpm}$, which is quite normal when the amount of steps taken is big. However, if the score provided by Algorithm 1 is 3 and the amount of steps taken is small, this may be an unusual behavior such as the red dots shown in Figure 6 at the end of the graph. These dots show that a high heart rate is detected while an intense activity is not performed by the user.

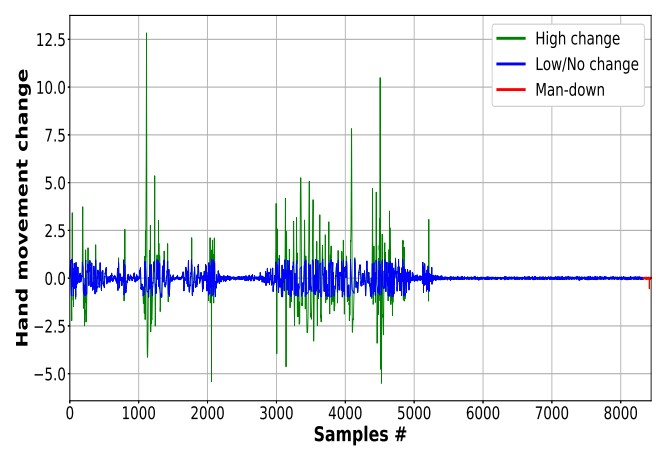

Fig. 5: Man-down state detection using motion change

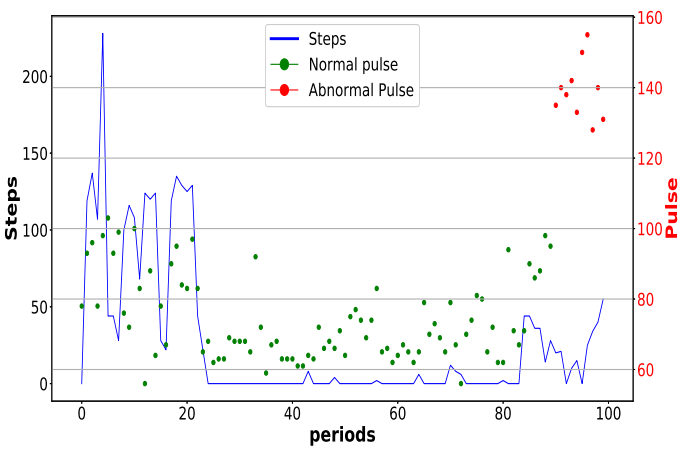

Fig. 6: Abnormal heart beats detection

Figure 7 shows the application that is implemented on the smart-watch. The user can use the button "Alert" to manually trigger one. Figure 8 shows the smart-phone application from the point of view of the receiver. An alert describing the emergency is illustrated in red at the top of the application, the location and a button that draws the direction to the "man down" are also provided.

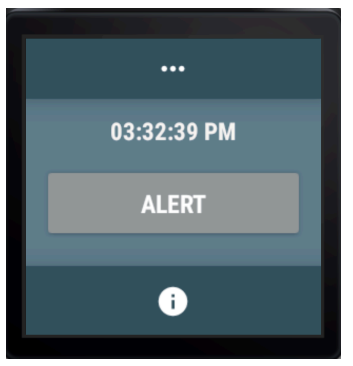

(a) Manual alert transmission

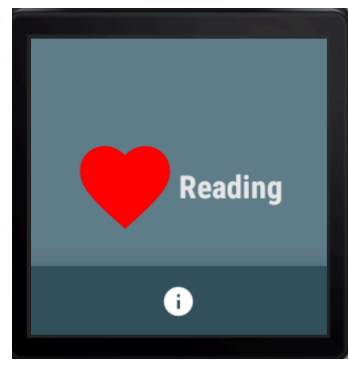

(b) Heart rate collection
Fig. 7: Captures from the wearable application

\section{A. Energy consumption}

In this section, an estimation of the operational lifetime of the system is calculated. Both the smart-watch and the IoT device are the main concerns since they are required to be active all the time.

We have measured the battery charge drainage in mAh of the IoT device using the measured current and the electrical 


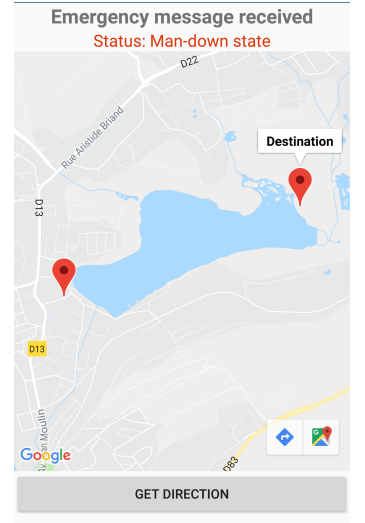

(a) Receiving new emergency alert

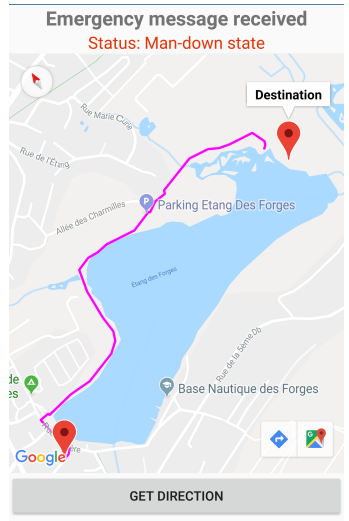

(b) Direction to the location of the sender
Fig. 8: Captures from the mobile application

power. It turned out that it is consuming on average $1 \mathrm{mAh}$ every 35 seconds as shown in Figure 9a. In our experiment, we have used a $3.7 \mathrm{~V}$ LiPo battery with a capacity of $800 \mathrm{mAh}$, which means the IoT device can operate for 7.7 hours.

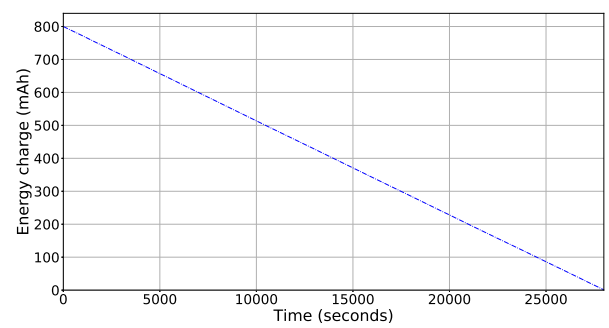

(a) IoT device battery charge drainage

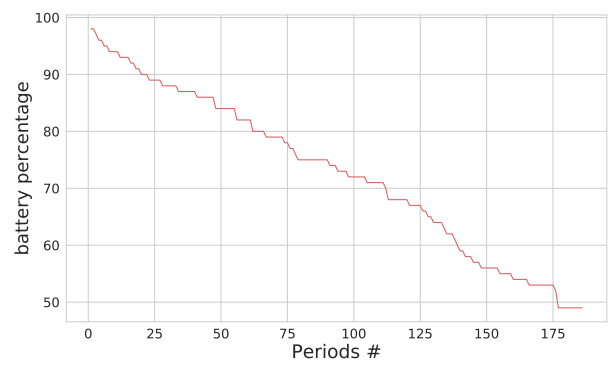

(b) Polar M600 battery level over 180 periods

Fig. 9: Energy consumption of the wearable devices

For the smart-watch, we ran the application where motion and pulse sensors are continuously collecting data while logging the battery level every 1 minute period. The graph in Figure 9b shows that it takes about 3 hours for the watch to reach half its battery life. Looking at these results we can conclude that the system can operate for $6 \mathrm{~h}$ approximately. In future work, we aim to optimize the algorithms and improve the energy efficiency of the devices by incorporating adaptive sampling techniques [12].

\section{CONCLUSION}

In this paper, we propose a decentralized LoRa based emergency alert system that can automatically identify and localize a victim in areas with no network coverage. The system is composed of a smart-watch that is used to monitor the heart rate and the activity of the wearer, a GPS enabled IoT device that transmits an alert via LoRa when triggered by the watch, and finally, a smart-phone that visualize the alert alongside the location and the direction to the victim. Our system has been deployed and tested in a remote area located in the city of Belfort in France. Both the activity detection and the heart rate monitoring algorithms have been validated. Moreover, successful transmission of an alert in case of emergency has been proven to work as intended.

\section{ACKNOWLEDGMENT}

This work is partially funded by the EIPHI Graduate School (contract "ANR-17-EURE-0002") and the PHC CEDRE Porjetct RESEAUCORP N: 40283YK.

\section{REFERENCES}

[1] F. Wu, J. Redout, and M. R. Yuce, "We-safe: A self-powered wearable iot sensor network for safety applications based on lora," IEEE Access, vol. 6, pp. 40 846-40 853, 2018.

[2] A. Mdhaffar, T. Chaari, K. Larbi, M. Jmaiel, and B. Freisleben, "Iotbased health monitoring via lorawan," in IEEE EUROCON 2017 -17th International Conference on Smart Technologies, July 2017, pp. 519524.

[3] F. Wu, T. Wu, and M. Yuce, "An internet-of-things (iot) network system for connected safety and health monitoring applications," Sensors, vol. 19, no. 1, p. 21, 2019.

[4] J. Petjjrvi, K. Mikhaylov, M. Hmlinen, and J. Iinatti, "Evaluation of lora lpwan technology for remote health and wellbeing monitoring," in 2016 10th International Symposium on Medical Information and Communication Technology (ISMICT), March 2016, pp. 1-5.

[5] "Two hikers found a message in a bottle and helped rescue a stranded family., september 2019." https://edition.cnn.com/2019/09/11/us/california-hikers-bottle-messagesearch-trnd, accessed: 2019-09-16.

[6] L. Schussman, L. Lutz, R. Shaw, and C. Bohnn, "The epidemiology of mountaineering and rock climbing accidents," Journal of Wilderness Medicine, vol. 1, no. 4, pp. 235-248, 1990.

[7] D. A. Lack, A. L. Sheets, J. M. Entin, and D. C. Christenson, "Rock climbing rescues: causes, injuries, and trends in boulder county, colorado," Wilderness \& environmental medicine, vol. 23, no. 3, pp. 223230, 2012.

[8] G. Bianchi, O. Brügger, and S. Niemann, "Skiing and snowboarding in switzerland: Trends in injury and fatality rates over time," in Snow Sports Trauma and Safety. Springer, Cham, 2017, pp. 29-39.

[9] J. Azar, C. Habib, R. Darazi, A. Makhoul, and J. Demerjian, "Using adaptive sampling and dwt lifting scheme for efficient data reduction in wireless body sensor networks," in 2018 14th International Conference on Wireless and Mobile Computing, Networking and Communications (WiMob), Oct 2018, pp. 1-8.

[10] "National early warning score (news) 2, royal college of physicians, london, u.k., december 2017.' https://www.rcplondon.ac.uk/projects/outputs/national-early-warningscore-news-2, accessed: 2019-08-27.

[11] "Average steps per minute for different exercises, july 2019." https://www.verywellfit.com/pedometer-step-equivalents-for-exercisesand-activities-3435742, accessed: 2019-08-28.

[12] G. B. Tayeh, A. Makhoul, D. Laiymani, and J. Demerjian, "A distributed real-time data prediction and adaptive sensing approach for wireless sensor networks," Pervasive and Mobile Computing, vol. 49, pp. 62-75, 2018. 